\title{
CONFERENGE PROGRAM
}

The Ninth International Conference on

\section{HUMAN ANTIBODIES \& HYBRIDOMAS}

\section{6-18 September 2002}

University Hospital, Berne, Switzerland

Sponsored by the International Journal HUMAN ANTIBODIES

In Association with The Rajko Medenica Research Foundation and Shantha Biotechnies PVT Lud.

Programme Chairman Mark Glassy (Phofores, RMRF, Sas Diega, USA)

Scientific Advisory Panel

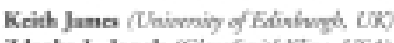

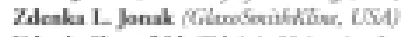

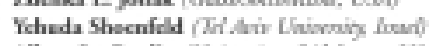

Albart Le Baplie (Chinmity of Alabine, LSW

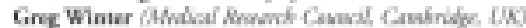

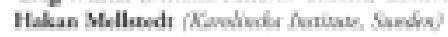

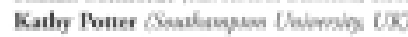

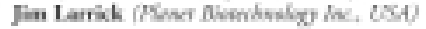

Laval Ongurist:

Aleis lang (BERNA ANOTECH, Suituntome)

Krower Sprohlor:

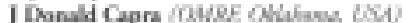

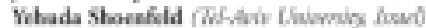

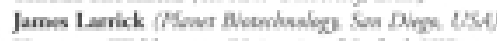

Hemaan Waldeans Chimenivy of Oagind UK.

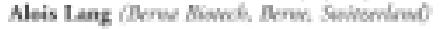

\section{CONFERENCE PROGRAM}

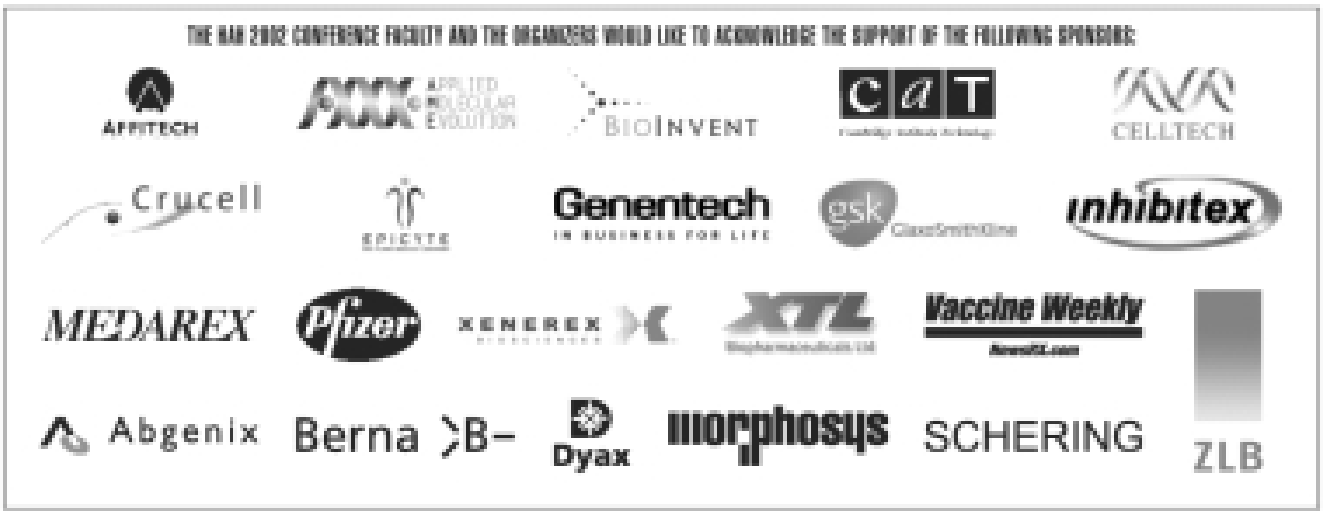

HAH 2002 Canference Secretariat, MFETINCS MANAGEMFNT

The Bam, Rale Meadow, Seation Lane, Milfoed, Sarney GUS 5MD, United Kingdom

Conference Manager Caraliac Sumaer Telephones $+44(0) 1433427770$ Faxt +44 (0) 1483426516

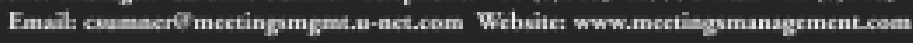




\section{CONFERENGE PROGRAM}

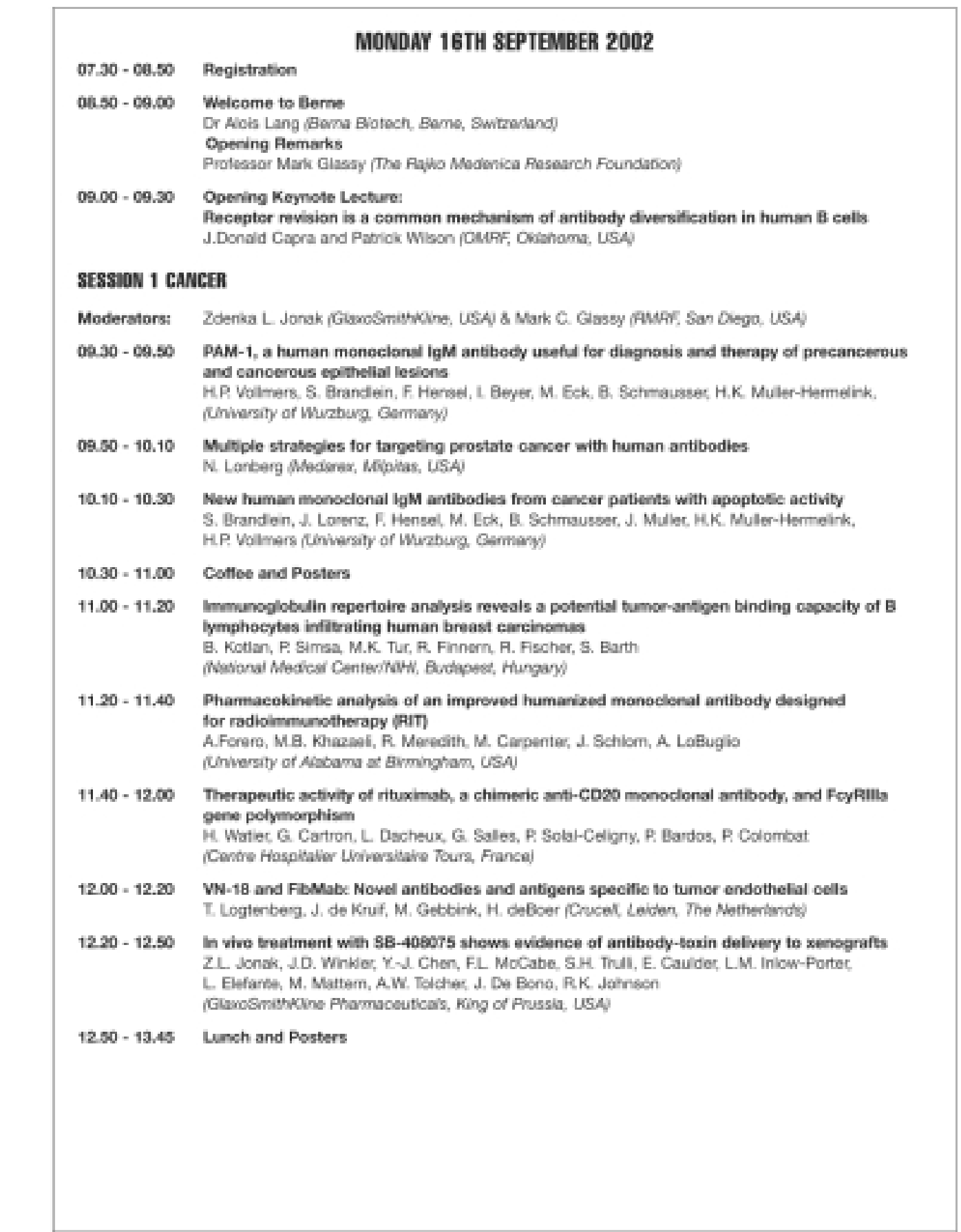

HaIt 2002 Conference Secretariat, MIETINGS MANMGEMENT

The Berm, Rake Medows, Searion Lane, Millford, Sarrey GUs SAD, United Kinglome Conference Menagen Ceroline Sumner Telephonet +ff (0) 1483427770 Fax +14 (0) 1483423516 Emalh coumaeremeetingsongas.a-nec.com Webshet www.mectingsmanagement.com 


\section{CONFERENGE PROGRAM}

\section{SESSION 2 AUTOIRMUNITY}

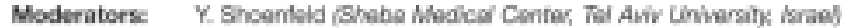
8 K. Potter (University of Southampton, tor)

13.45 - 14.15 Kernote Lecture:

The infectious origin of A.PS. Molecular mimicry between microbial pathogens and B2-C.PI: The induction of experimental APS

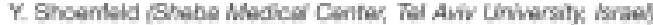

14.15 - 14.35 Production of human monoclonal antibodies to desmoglein 3 from Pemphagus vulgaris patient K.C. Bhol, A.R. Ahmed (Havad School of Dantal Modicina, Boaton, USA)

14.35 - 14.55 The mosale of autoimmunity The role of environmental factors in autoimmune diseases Kohuda Shoendald (Shabe Medical Conter, Tel Awi Lhiversity, Israel)

$14.55-15.15$ Similar patterns of 8 cel perturbation are found in the peripheral blood from patients with systemic hupus erythematosus (SLE) and infectious mononucleosis (IM)

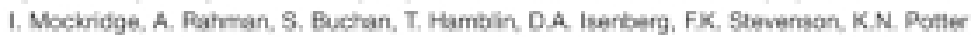
Tenovis Laborafory. Unversity of Southampton, UNo

15.15- 15.45 Tea and Posters

15.45 = 15.05 Search for a monoclonal antibody mimiokng naturally ocourring anti-C.3/C.3b antbodies that regulate cemplement amplification

H.U. Lutz, M. Schlumberger. P.J. Spath, E. Jelezarova, A. B Lang

(ETH Zunich/ZLE Bloplasme LravBornaBlatoch, Suitaertand)

16.05 - 16.25 Comparablity testing of a recombinant human anti-Ph D antbody derived from two ditferent cell lines using biophysical and biochemical parameters? relevance for scale up manufacturing H.d. Krieg S. Schurch, J. Schaler, S. Dejardin, A. Tu, S. Miescher, P.A. Gred, N. Mamod,

H. Amstutz (inseispital Bevne. Swizeriand)

16.25 - 16.45 Anti-daDNA anti-idiotypic activity as the main mechanism of IVIC effect in SLE L. Pauovs. B. Giburd, M. Blark, L. Goldbeng. J. Kopolowic, M. Ehrenteld. J. Povenshy, Y. Shoenfeld (Sheba Modical Cendec Dow Awv Lhiversity Isoet)

16.4S - 17.05 Functional properties of anti-0 monoclonal antibodies; role of host cell expression system and in vitro correlstion between ADCC and 122 release from Jurkat CD16

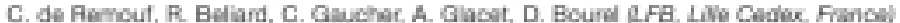

$17.05-17.25$ In vitro functional assessment of a recombinant anti-Ah 0 antibody relevance to clinical performance

H. Amstutz, S. Dejardin, T. IH, M. Soyctiec A. Holmern, S. Nahroang B. Stader S. Miescher P Lerch Ansolspital, Berne, Switsertand

17.25 - 18.05 Kermote Lecturs:

If we can make any antibody we want then how come we don't have it? Jamea Lamick P Planot Elodechnologx, San Diago, USA.

Evening Welcome Reception (Time to be confirmed) 


\section{CONFERENGE PROGRAM}

\begin{tabular}{|c|c|}
\hline \multicolumn{2}{|r|}{ TUESDAY 17TH SEPTEMBER 2002} \\
\hline $08.30-09.00$ & $\begin{array}{l}\text { Keynote Lecture: } \\
\text { Stealth antibodies } \\
\text { Hermann Waldmann (University of Contord, ung }\end{array}$ \\
\hline Moderstor: & Keith James (Eolvourght) \\
\hline \multicolumn{2}{|c|}{ SESSION 3 EUERETE TECHNOLOGIES } \\
\hline Moderator: & Keith James (Edinburgh) \\
\hline $0900=09.20$ & $\begin{array}{l}\text { Selection and engineering of antibodies for human therapy } \\
\text { D. Atwal (Cedtech Chirosciences. Slough, UK) }\end{array}$ \\
\hline $00.20-00.40$ & $\begin{array}{l}\text { The n-CoDeR TM antibody libraraies: concept and applications } \\
\text { E. Soderind (Blainvent Therapeutics } M B, \text { Lund, Swedery }\end{array}$ \\
\hline $09.40-10.00$ & 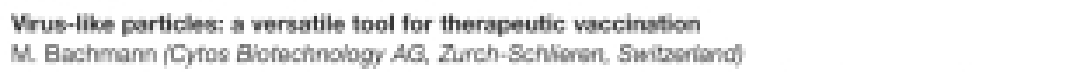 \\
\hline $1000=10.20$ & $\begin{array}{l}\text { Genetio immunization: a rapid way of developing high quality disgnostic or therapeutio } \\
\text { antbodies agsinst native proteins } \\
\text { J. Thompson (GENOVAC AG, Frobug, Gormany) }\end{array}$ \\
\hline $10.20=10.40$ & 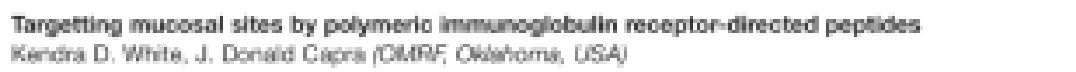 \\
\hline $10.40-11.00$ & Coflee and Posters \\
\hline \multicolumn{2}{|c|}{ SESSION 4 MOLECULAR BIOLOGY (I) } \\
\hline Moderstors: & 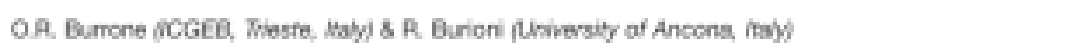 \\
\hline $11.00-11.30$ & $\begin{array}{l}\text { Tumor rejection in anti-idiotypic DNA vaccination is antibedy-mediated } \\
\text { M.C. Gaspere, F. Bervenuti, O.F. Burrone fCGEB, Theste, frayy }\end{array}$ \\
\hline $11.30-12.00$ & 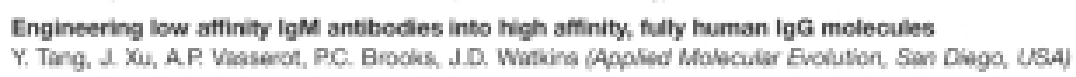 \\
\hline $12.00-12.30$ & 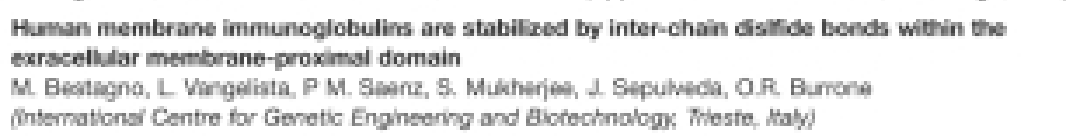 \\
\hline $12.30-13.00$ & 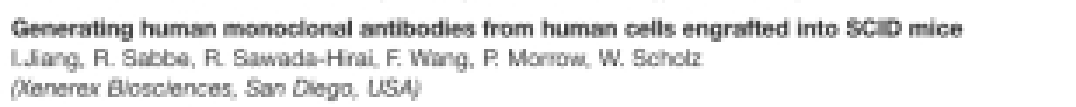 \\
\hline $1300-14,00$ & Lunch and Posters \\
\hline \multicolumn{2}{|c|}{ SESSHON 5 MOLEEULAR BIOLOGY (II) } \\
\hline Moderators: & 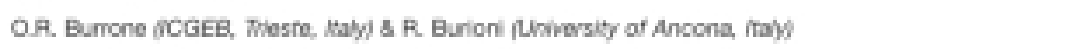 \\
\hline $14.00-14.20$ & $\begin{array}{l}\text { Deimmunised immunotoxins for multiple targets } \\
\text { F. Caer (Biovision L.tul, Aberdaen, LWS }\end{array}$ \\
\hline $14.20 * 14.40$ & $\begin{array}{l}\text { From EST to IHC - human amtibodies for target validation and drug development } \\
\text { K. Watzka Morphosys AG, Morthereid, Germsyy) }\end{array}$ \\
\hline $14.40-15.00$ & $\begin{array}{l}\text { Selecting and optimizing therapeutic antbody lewds } \\
\text { C.P. Shomock, L. Jermubus (Cambridige Antbody Tochnology, uap }\end{array}$ \\
\hline $15.00-15.20$ & $\begin{array}{l}\text { Troybodies", a novel approach to cancer vacoines } \\
\text { O.H. Brikke, L. Sindie. B. Begen (Awtech AS, Osb, Narwey) }\end{array}$ \\
\hline $15.20=15.40$ & $\begin{array}{l}\text { Affinity maturation of human Fab antibedies by yeast display } \\
\text { S.E. Hufton (Dyar SA. Bobjum) }\end{array}$ \\
\hline $15.40-16.20$ & Cottee Break (Extended for Posters) \\
\hline
\end{tabular}

HAH 2002 Conference Socreariat, MEETINGS MANAGEMINT

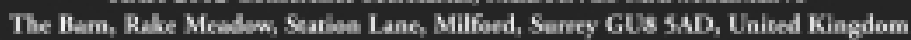
Conference Managen Caroline Sumner Telephones +44 (0) 1483427770 Fexi +4i (0) 1485425516

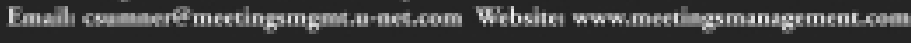




\section{CONFERENGE PROGRAM}

\section{SESSION 6 POSTER SESSION}

\section{SESSION 7 MOLFELAR BIOLOGY (III)}

Moderators: To be confirmed

16.20 - 16.40 Cloning and expression of a non-smal cell lung canes specific RM2 scfv in Pichie pestoris P. Waichmoker, M. Pari, K. Kota, S. Chekrabarti, S. Mukerjee (Shantha Biolechries PVT Lid, Hyderabad, India)

16.40 - $17.00 \quad$ Human recombinant autountbodies speciflo for the La (SS-B) autosntigen from patient derhed phage display combinatorial antibody libraries

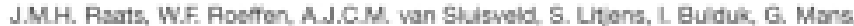

F.H.J. Vin den Heogen, G.J.M. Pruin. W.J. van Venreai Rhiveraity of Nymegen, The Netherisnds:

17.00 - 17.20 Production and characterization of monoclonal antibodies produced in the human cell line PER.C6 0. Jones, N. Kroos, R. Anems, N. van Oik. H. Kaptern, R. Zwiven, T. Logterberg, A. Bout (Cucel, Leiden, The Nistherlanda)

$17.20-17.40$ Biological acthily of human 'condtionsl' anti-Fcepla-chain antibodes is defined by their light chain M.Fux, T. Bobrrynski, M. Vogel, J. Pachlogrik, M. Horn. B.M. Stader, S.M. Meschar

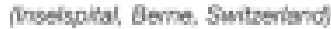

17.40 - 18.00 Human miniantibodies against erthopexviruses A.A. lyicher ef s. MECTOA. Novosibirst. Russia)

1800 - 18.20 Using XenoMouse" mice for gernerating optimal haman antibodies for therapeutic leads L.L. Green (Abganix mc., Fremont, Cavtomia, USA)

Evening: Conderence Dinner (Time to be confirmed)

\section{WEDNESDAY 18TH SEPTEMBER 2002}

\section{SESSION 8 INFECTIOUS DISEASES}

Moderator: Alois Lang (Bowna Blotoch Lad, Bovne, Suntzontand)

09.00-09.30 Keynote Lecture:

Status of human monocional antibody therapy against gram-negative pathogers

A. Lang (Bemabiatech Berne. Surtaentand

00.30 - 10.00 Moleculer dissection of the anti-HCV humaral respense by phage display reperteire clening A. Burioni Rhiversity of Ancors, Italy?

10.00 - 10.30 Human monocional antbedies specific for conformation-sensitive epitopes of V3 mediate cross-clade neutralization of HN-1 primary isolates

M.K. Gorry, C. Wilims, B. Volskx K. Pervesz, S. Cohen, V. Polonis, W \&. Honran, C. Krachmarev, A. Pinter S. Zolla Pasner plow York Chiveraty School of Mockicine, USA

$10.30-11.00$ Cotlee and Posters

$11.00-11.20$ Aurexis"w, a humarised monoclonal antibody protective against S. aurous infection J.T. Hutchins, D. Brysnt, P. Domanski, L. Donald, A. Hal, P. Potel, B. Prater. J. Pobbins. P. Syribeys,

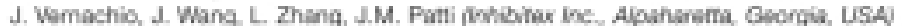

11.20 - 11.40 Generation of fully human monoclonal antibodies speoifio to bscterial and fungal pathogens in a humanimosise racliation chimera: the Trimera system

F. Eren, S. Dagan et al. PCTL. Blophamuceuticals Lfd, Fehovot, Isved

11.40 - 12.00 Muman T hymphotropic virus type 1 (HTLV-1) audoantibodies and type 1 diabetes mellitus in Jamaicans

M. Fisher Smikle finiversity of the West indies, Kingston, Jamaical

$12.00-12.20$ Clinical evaluation (Phase I) of a combination of two human monecional antibedies to HBV: Sstety and antiviral properties

A. Eren, S. Dagan ot al. PCTL. Bigohamacouticals Lfd, Fohovot, havelf

12.20 - 12.30 Closimg remarks and Imitation to attend HAH 2009 in Japan Prat M.C. Glasey PHAH 2002 Conterence Chaimany

HIM 2002 Conference Secreariar, MIETINGS MANMGEMINT

The Barn, Ralae Meadow, Sarioe Lane, Milifoed, Surrey GUa SAD, Unired Kingdem

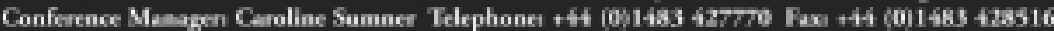
Emall caumaeremeetingsmgac.s-nec.cen- Webshet www.mectingsmanagement.com 


\section{POSTER PROGRAM}

Postres:

Anchitactura features of hurnan lag that nodulate its catabels

nekithe in the nat

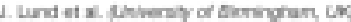

posten 2

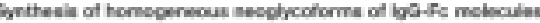

and herr Amosonal antertien

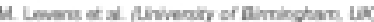

posten a

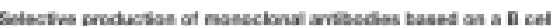

Gepetro tochenion

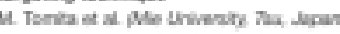

postue 4

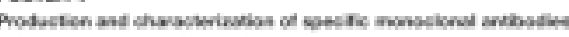

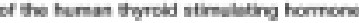

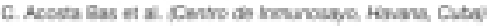

\section{posters 5}

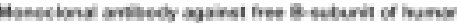

chorionic gonadorophin

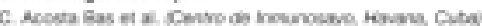

\section{DOSTLR}

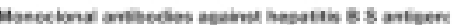

produston, characieriestion, and une for thoonati.

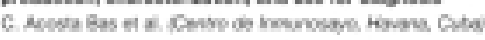

postre?

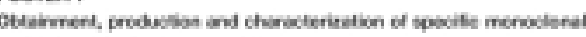
artibody of the heman trypain

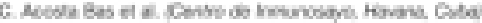

\section{POSTERA}

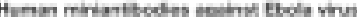

S. Thermosa et a. MECTOR Navedaink Austial

\section{posters 9}

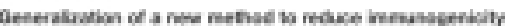

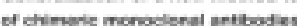

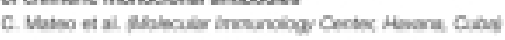

poster 90

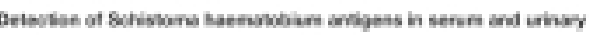
tract thase of infected hamuten uning apecise monoclonal antitedy

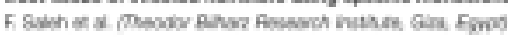

POSTER it

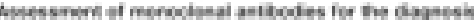

of Schintsosma hoerratatium infecten

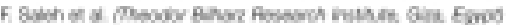

POSTRR 9

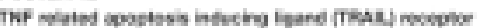

artibody aporinta

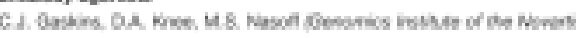
Neawerot Foundason, Sin Dinga, USA)

poster so

Production af varibus huran menocianal antibodes

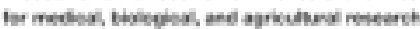

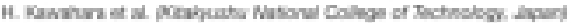

posten s4

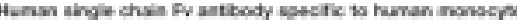

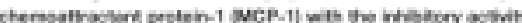

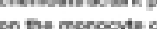

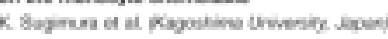

pesten 15

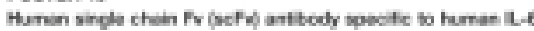

whin the mithory acteity to ll-6 signating

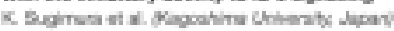

PCETER 16

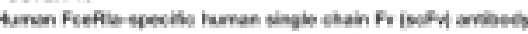

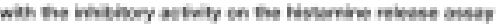

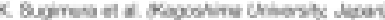

postra is

Dovelagenam of noved antigan binders bated on threriation of Vis somaina

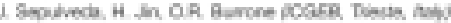

posten is

Moconthant anthodke in encer beatrom:

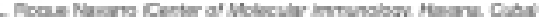

POGTER 19

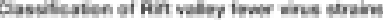

asine moneseral antlextes

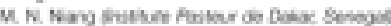

posten 20

mennascintioraphy of eaperimertal turnar medivis vaing

MnTe labeted momostered arthosles.

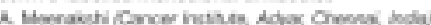

eosten z1

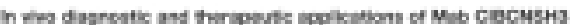

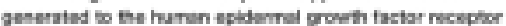

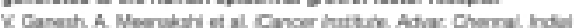

\section{HeबrEA 20}

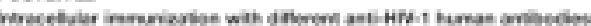

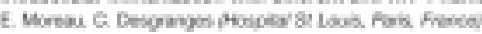

\section{posters as}

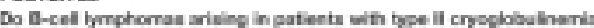

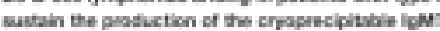

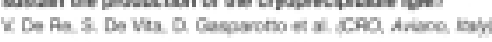

resters at

the mxiphe of immunogletulin variate negiom of menoclonal

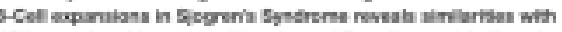

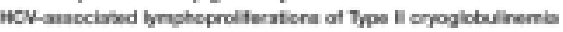

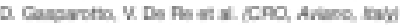

\section{Festen as}

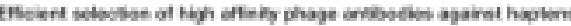

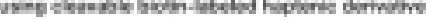

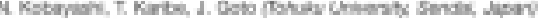

PCGTER $2 x$

dereskertos and valdation of nowed targets ansecibed wich

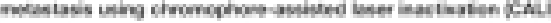

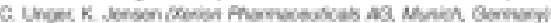

posten 21

Preperation of the mouse and human chineric aritbedies

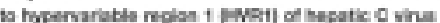

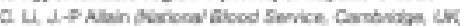

\section{Fegren as}

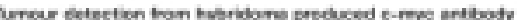

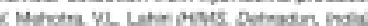

PCGTrR 28

High athiancy creatien et hemen monsciond

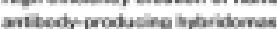

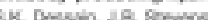

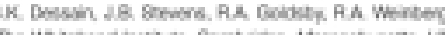

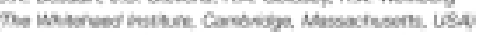

